\title{
Optogenetic Activation of the Excitatory Neurons Expressing CaMKII $\alpha$ in the Ventral Tegmental Area Upregulates the Locomotor Activity of Free Behaving Rats
}

\author{
Songchao Guo, ${ }^{1}$ Sicong Chen, ${ }^{2}$ Qiaosheng Zhang,, ${ }^{1}$ Yueming Wang, \\ Kedi $\mathrm{Xu},{ }^{1}$ and Xiaoxiang Zheng ${ }^{1,2}$ \\ ${ }^{1}$ Qiushi Academy for Advanced Studies, Zhejiang University, Hangzhou 310027, China \\ ${ }^{2}$ Institute of Biomedical Engineering, Zhejiang University, Hangzhou 310027, China \\ Correspondence should be addressed to Kedi Xu; xukd@zju.edu.cn
}

Received 4 December 2013; Accepted 14 January 2014; Published 10 March 2014

Academic Editor: Ting Zhao

Copyright (c) 2014 Songchao Guo et al. This is an open access article distributed under the Creative Commons Attribution License, which permits unrestricted use, distribution, and reproduction in any medium, provided the original work is properly cited.

The ventral tegmental area (VTA) plays an important role in motivation and motor activity of mammals. Previous studies have reported that electrical stimulations of the VTA's neuronal projections were able to upregulate the locomotor activity of behaving rats. However, which types of neurons in the VTA that take part in the activation remain elusive. In this paper we employed optogenetic technique to selectively activate the excitatory neurons expressing CaMKII $\alpha$ in the VTA region and induced a higher locomotor activity for free behaving rats. Further behavioral studies indicated that reward learning mediated in the enhancement of the rat locomotor activity. Finally the immunohistochemistry studies explored that the excitatory neurons under the optogenetic activation in VTA were partly dopaminergic that may participate as a vital role in the optogenetic activation of the locomotor activity. In total, our study provided an optogenetic approach to selectively upregulate the locomotor activity of free behaving rats, thus facilitating both neuroscience researches and neural engineering such as animal robotics in the future.

\section{Introduction}

Optogenetics comprises a set of techniques that integrate the opsin genes into specific types of neurons to selectively probe the neural circuits $[1,2]$ and has currently been introduced into a growing number of neuroscience researches [3-6] and neural engineering systems such as the brain-machine interfaces $[7,8]$. The optogenetic technique enables either excitation or inhibition of selected neural populations under the delivery of light at specific wavelengths $[9,10]$. Basically the opsin genes are able to express light-sensitive membrane ion channels, produce ion flows, and thus induce or suppress the action potentials in living neural populations [9]. The channelrhodopsin-2 (ChR2), one of the opsin cation channels $[10,11]$, is typically transduced into excitatory neurons $[12,13]$ under the guidance of certain promoters such as the calciummodulin dependent kinase II type- $\alpha$ (CaMKII $\alpha)$ promoters.
The specific neurons with ChR2 and CaMKII $\alpha$ expressions would produce action potentials upon the delivery of blue light at a central wavelength of $473 \mathrm{~nm}[1,2]$.

In animal brains, the vast majority of the excitatory neurons expressing CaMKII $\alpha$ are distributed in cortex areas and hippocampus $[1,14]$. For rats, the CaMKII $\alpha$ was also found in deep brain regions, for example, the ventral tegmental area (VTA) within the ventral striatum $[15,16]$. The VTA comprises a variety of neurons located on the floor of the midbrain that relate to the mesolimbic dopaminergic system and is widely implicated in the natural reward circuitry of the brain as well as drug addiction and motor activity. Particularly, electrical stimulations of the medial forebrain bundle (MFB), one of the neuronal projections from the VTA region, were able to upregulate the rat locomotor activity as a part of the rat-robot systems $[17,18]$. However, the precise mechanisms underlying such rat-robot systems remain unclear, largely due 
to the extensive and unselective effect of electrical stimulation on all types of neurons. Actually, besides dopaminergic neurons, the VTA also contains glutamatergic neurons [19] that are regarded as a type of excitatory neurons expressing CaMKII $\alpha$. Yet few researches have been engaged in how such excitatory neurons take part in the VTA neuronal activities and whether these neurons alone could influence behavioral conditionings such as locomotor activities.

In this paper we selectively activated the excitatory neurons in the VTA region by optogenetic transductions of the CaMKII $\alpha$-ChR2-mCherry virus into the rat brain. Both histological and electrophysiological methods verified the robustness of the optogenetic manipulations. We found that the optical activations on these VTA neurons were able to induce an enhanced locomotor activity of free roaming rats. Further results revealed that these VTA neurons under optogenetic activation may involve the reward learning mechanisms and were partly dopaminergic, consistent with previous reports on both structural and functional properties of the VTA [20-24]. Taken together, our study based on the optogenetic techniques has provided a novel method to selectively upregulate the locomotor activity of free behaving rats, which could be integrated into the rat-robot systems for precise controls. Also we have further approached the explanation of mechanisms underlying the rat-robot control strategies, thus facilitating future work on both animal robotics and neuroscience researches.

\section{Material and Methods}

2.1. Animal Subjects. Adult male Sprague-Dawley rats with $180 \pm 10$ g body weight were chosen from Zhejiang Academy of Medical Sciences (Hangzhou, China). All the rats were housed in a temperature-controlled room $\left(23 \pm 3^{\circ} \mathrm{C}\right)$ with access to water and mildly food-deprived to $85 \%$ of freefeeding body weight. The rats were kept in individual cages on a $12 \mathrm{~h}$ light/dark cycle (lights on at $6 \mathrm{am}$ ).

\subsection{Surgeries}

2.2.1. Optrode Implantation. The optrode array device used in this paper was designed in our previous study [25]. The implantation of the array device was implemented by craniotomy on the rats over four postnatal weeks. In general the rats were anesthetized with $1.0 \%$ sodium pentobarbital, sheared over the head, and fixed in a stereotaxic apparatus (Stoelting Co., Ltd., USA). For each subject, the craniotomy was centered on the brain region dorsal to the left VTA region: $-4.8 \mathrm{~mm}$ posterior to the bregma (AP), $1.0 \mathrm{~mm}$ lateral to the midline $(\mathrm{ML})$, and $-8.0 \mathrm{~mm}$ ventral to the cortical surface (DV) according to the atlas of Paxinos and Watson. A series of skull holes were drilled for placement of four skull screws to provide mechanical support as well as common ground references for the implanted array device. In particular, a round craniotomy window with $1.0 \mathrm{~mm}$ diameter was drilled for implanting the array device. The dura matter was carefully peeled away using a sterilized needle with operations under microscopy. Then the array device, yet without the optical fiber, was stereotaxically implanted through the craniotomy window into the targeted brain area exactly above the left VTA region. In addition, a ground reference electrode from the array device was bundled firmly on all the skull screws. Finally the craniotomy window was filled with clinical ionized gel for protecting the inner brain and the entire scalp area was covered up using dental acrylic. The rats were allowed to recover for 5-7 days before use in the viral delivery and the optical fiber implantation.

2.2.2. Viral Delivery and Fiber Implantation. The surgeries for viral delivery and optical fiber implantation were implemented via the optrode array device as described above. The rats were anesthetized with $1.0 \%$ sodium pentobarbital and again stereotaxically fixed. Adenoassociated viral vector serotype $5(\mathrm{AAV}-5)$ carrying the opsin gene of ChR2 and the gene for red fluorescent protein (mCherry) under CaMKII type- $\alpha$ promoter (Figure 1(a), AAV-CaMKII $\alpha$-ChR2-mCherry, $\sim 5 \times 10^{12}$ titer, Neuron Biotech, Shanghai, China) was delivered into the rat brain using the microinjector (World Precision Instruments, Co., Ltd.) fixed on the stereotaxic apparatus. For each rat, $1.0 \mu \mathrm{L}$ of viral vector was injected through the guide cannula of the optrode array device into the left VTA (DV $=-8.5 \mathrm{~mm}$ ). The timing procedure of the viral injection was consistent with [11]. The microinjector and surgical apparatus were thoroughly sterilized after viral injection.

Upon finishing the viral delivery, an optical fiber with one-end FC tail was stereotaxically implanted through the guide cannula such that the fiber tip reached the dorsal edge of the VTA region ( $\mathrm{DV}=-8.0 \mathrm{~mm}, 0.5 \mathrm{~mm}$ upper than the viral injection site) and the FC tail lay above the optrode cannula. Finally the optical fiber was covered up and mounted firmly on the entire array device using dental acrylic. The rats were kept for recovery and ChR2 expression for four weeks.

2.3. In Vivo Optical Stimulation and Electrophysiology. The optical instruments consisted of a $500 \mathrm{~mW}$ laser emitting $473 \mathrm{~nm}$ blue light (BL473T5-320FC, Shanghai Laser \& Optics Century Inc., China) and a 3-meter optical fiber jumper with 50/125 multimodal glass optical fibers inside. The fiber jumper was connected to the laser and coupled to the optical fiber on the rat head by a plastic, tube-shaped FC-FC interface adapter [25]. The laser was triggered by transistortransistor logic (TTL) pulses generated from a PG4000A digital stimulator (Cygnus Technology Inc., USA). The light power was measured by an optical power meter (LTE-1A, Chinese Academy of Sciences, Beijing, China). Usually a light power of $1-3 \mathrm{~mW}$ at the end of optical fiber jumper was approved for the following in vivo studies.

For in vivo electrophysiology, the electrical signals were recorded from the free behaving ChR2 rats via the Omnetics connector [25] jointed on the optrode device over the rat head. The signals were processed by a Plexon Multichannel Acquisition Processor (1-40 kHz rate, Plexon Inc., Dallas, TX). Signal preprocessing, including amplifications on programmable gain and filtering (set at $150 \mathrm{~Hz}-8 \mathrm{kHz}$ band pass) for spikes, was implemented using the OmniPlex Controller 


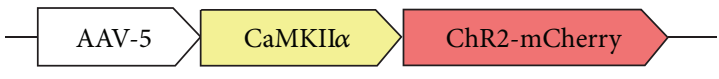

(a)

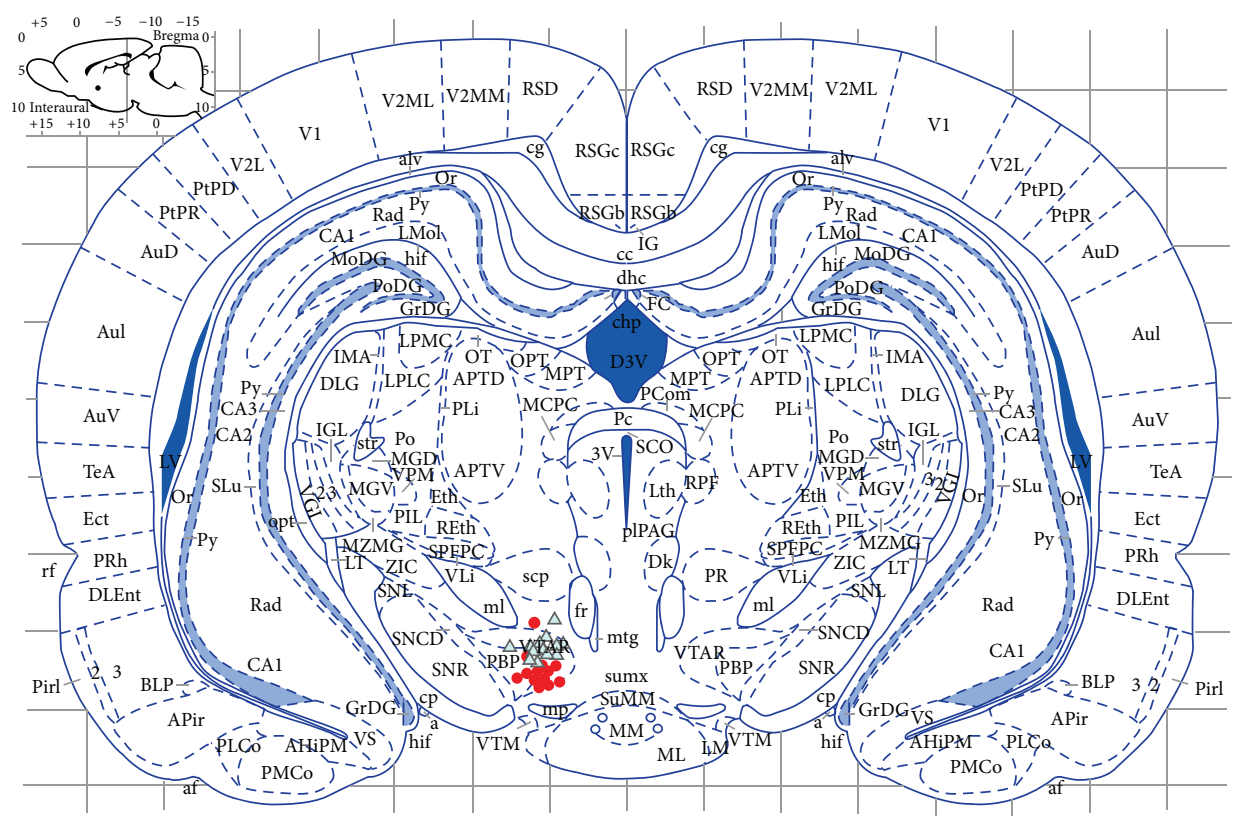

(b)

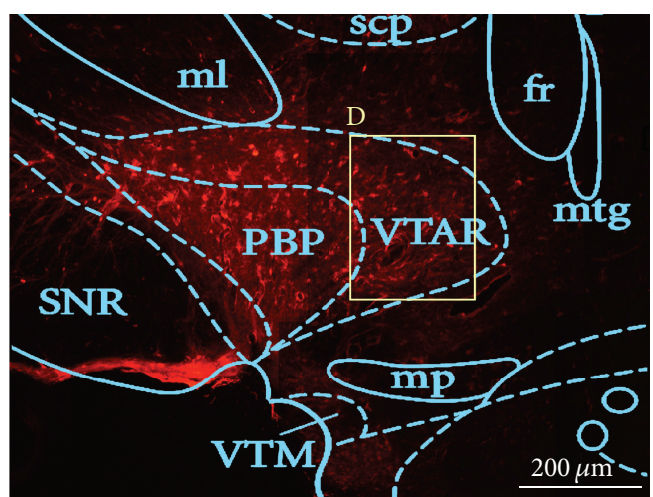

(c)

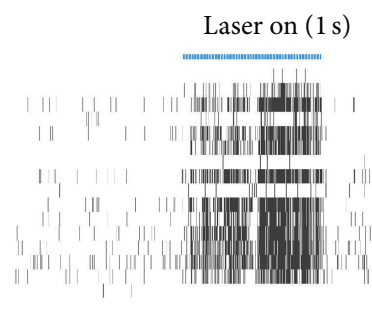

(e)

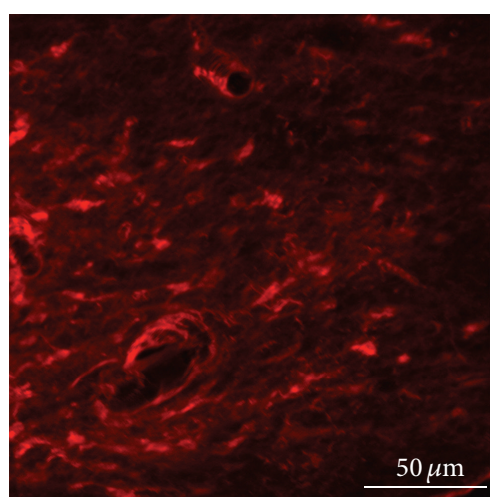

(d)

Laser on $(1 \mathrm{~s})$

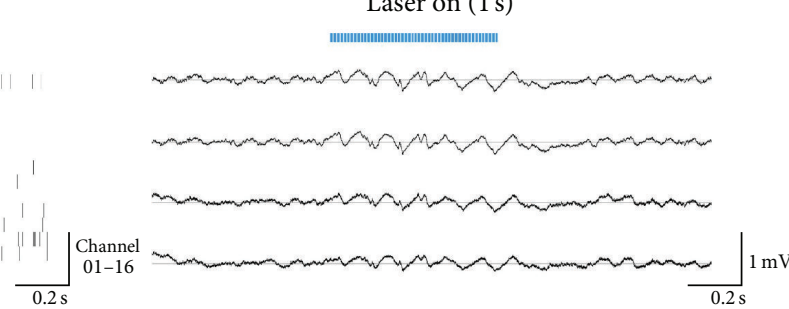

(f)

FIGURE 1: Histology and in vivo electrophysiology for verification of the optogenetic studies. (a) Sketch of the AAV vector used for the optogenetic transductions in this study. (b) The centers of viral-delivery regions (the red circular dots) and the placements of optical fiber tips (the blue triangular dots) for all the optogenetic rats $(n=6$ for the free-moving tasks, $n=6$ for the lever-pressing tasks, and $n=2$ for erroneous displacements). These two series of dots were measured by individually locating the traces of optrode fiber tips and of the microinjector observed under microscopy. Both series of traces were measured in the targeted brain slices with AP $=-4.8 \mathrm{~mm}$ from Bregma and overlapped into the atlas of Paxinos and Watson. (c) A typical view of the ChR2-mCherry expressions on the neurons expressing CaMKII $\alpha$ in and around the brain region of VTA that was overlapped with the brain atlas. (d) An inner set of (c) obtained from the region of interest (ROI) from the VTA region (including VTAR and PBP). ((e)-(f)) In vivo electrophysiology recorded from the implanted optrode device. The blue bar shown in both figures represents a shot of laser stimulations with $15 \mathrm{~ms}$ pulse width, $50 \mathrm{~Hz}$ frequency, $1.0 \mathrm{~s}$ duration, and a light power around $1 \mathrm{~mW}$. (e) presents spike activities from all the sixteen channels of the optrode device where each short straight bar represents one spike firing, and (f) is the LFP signals recorded from four typical channels of the optrode device. 
as a part of the Plexon platform. Local field potentials (LFPs) were also recorded with band pass filtering at $10-170 \mathrm{~Hz}$ and digitized at $1 \mathrm{kHz}$ sampling rate.

\subsection{Design of Behavioral Experiments}

2.4.1. Free-Roaming Tasks with Field Tracking. The optogenetic rats were taken a series of behavioral experiments for free roaming in a circular field with 1.2 meters in diameter and were video-tracked throughout the experiments. The freeroaming tasks were given for 6 consecutive days. During each day, the rats were given three individual sessions, denoted as the nonstim, optic stim, and optic blocking sessions. For each session, the rats were allowed for 15 minutes free behaving to adapt the environment in the field with the optical fiber connected on its head, followed with a 10-minute test session: for the nonstim session, no laser stimulus was given; for the optic stim session, the rat was given a series of laser stimuli once every 0.6 second during the entire $10 \mathrm{~min}$ session with $15 \mathrm{~ms}$ pulse width, $50 \mathrm{~Hz}$ frequency, $0.2 \mathrm{~s}$ duration, and a light power at 1-3 $\mathrm{mW}$; yet for the optic blocking session, the same laser stimuli were given, but the laser was blocked on its path at the FC interface over the rat head (using a thin ceramic chip). The rat movements were video-recorded across the whole test session for further analyses.

The video recorded from each session was processed for rat figure recognition using the OpenCV techniques [26] such that the rat locations during the test session were captured as the planar coordinate data $(x, y)$ for the rat's gravity center. The data were then traced and jointed together to form a tracking map for free roaming in the field throughout the session (by MATLAB version 6.5, Mathwork software, USA), and the total intrasession distance of the free roaming rat was then calculated.

2.4.2. Lever-Pressing Tasks. The lever-pressing tasks involved two groups of rats: the optogenetic rats $(n=6)$ with ChR2 expression in the VTA region and the control rats $(n=6)$ with microinjection of saline instead of the viral vector. The lever-pressing tasks were conducted for 6 consecutive days with a 30-minute test session per day for each rat. During the test session, the rat again connected with the optical fiber on its head was gently placed into the chamber for lever pressing with a fixed-ratio 1 (FR1) schedule: upon one lever press, the lever would automatically trigger the PG4000A stimulator and the laser device to generate a 1.0-second laser stimulus with the same pattern as in the free-roaming tasks. A video camera was mounted on the top for video recording. Basically, the rat pressing the lever with its forepaws was judged as a "correct" or an "active" press; the lever pressing or hitting by other parts of the rat body was regarded as an "incorrect" or "passive" press. Both the numbers of correct and incorrect lever presses were individually counted.

2.5. Histological Studies. The rats after the behavioral experiments were anesthetized with a lethal dose $(240 \mathrm{mg} / \mathrm{kg})$ of sodium pentobarbital and perfused transcardially with $300 \mathrm{~mL}$ saline at room temperature (RT) followed by $300 \mathrm{~mL}$
$4 \%$ paraformaldehyde (PFA). The head was removed following perfusion and embedded in $4 \%$ formaldehyde solution for two days at $4^{\circ} \mathrm{C}$ fridge. The brains were then transferred into $30 \%$ sucrose and embedded for one day before the frozen section. The brains were sectioned into $40 \mu \mathrm{m}$ coronal slices. The frozen brain slices were used for verification of the recording and optical stimulating sites by observing the different optrode traces inside the slices under a microscope. Fluorescent images were taken to identify the distribution of ChR2-mCherry expression in and around the left VTA region. Two sets of traces on the brain slices, one was the centers of viral delivery and the other was the tips of optrode fibers, were individually measured with the assistance of the atlas of Paxinos and Watson for neuroanatomical studies.

Immunohistochemistry was conducted with the $40 \mu \mathrm{m}$ coronal brain slices prepared in the same manner. The brain sections were rehydrated by free-floating in the phosphate buffer solution (PBS) for 15 minutes. Tissues were next incubated in $-20^{\circ} \mathrm{C}$ pure methanol for 10 minutes. Thereafter the brain slices were incubated with the primary antibody (Rat antityrosine hydroxylase, Ab6211, Abcam, Cambridge, MA, USA) at a concentration of 1:500 diluted with $0.1 \%$ Triton X-100 and $4 \%$ Bovine serum albumin (BSA) in $4^{\circ} \mathrm{C}$ fridge over two days. After incubation with the primary antibody, the brain slices were then washed with PBS for $10 \mathrm{~min} \times 5$ times. The secondary antibodies (Alexa Fluor 488, rabbit anti guinea pig, Invitrogen, Carlsbad, CA, USA) at a concentration of $1: 1000$ in PBS were employed and the tissues were incubated for 2 hours at RT. Then the washing steps for the primary antibodies were repeated. The brain tissues were then carefully mounted on glass slides for later observations with confocal microscopy. For each optogenetic rat, part of the sections containing optrode traces was selected for detail analyses.

2.6. Data Analysis. Data acquired from the free-roaming studies were analyzed with two-way repeated measures ANOVAs, while Student's $t$-test was employed to compare the average distances across 6 sessions under the nonstim, the optic blocking, and the optic stim as well as the trained optic stim patterns individually, where $P<0.05$ indicated statistical differences.

For the lever-pressing tasks, numbers of lever pressing during one 30-minute session for both optogenetic rats and control rats were individually averaged for each day and were mapped to time-course curves. The data of both groups for the six days were individually compared again using $t$-test with $P<0.05$ indicating statistical differences.

\section{Results}

3.1. Optogenetic Expression and Optical Activation of Neural Activities in the VTA Region. For optogenetic transductions, the AAV-type-5 viral vectors carrying CaMKII $\alpha$-ChR2mCherry (see Figure 1(a)) were injected into the VTA region of the rat brains. Figures $1(\mathrm{c})$ and $1(\mathrm{~d})$ provided typical views of the histological data, which revealed a high density of ChR2-mCherry expressions in the targeted VTA region 
(VTAR and PBP) with normal cell morphologies. The center of the opsin expressions (red dots in Figure 1(b)) and the location of the optical fiber end (blue dots in Figure 1(b)) of each rat were presented on the brain slices as shown in Figure 1(b). The assembles of trace labels revealed that the ChR2 optogenetic expressions were precisely located in the VTA region with the optrode fiber tip $\sim 0.5 \mathrm{~mm}$ above for most of the rats, appropriate for light penetrating on the brain regions with opsin expressions.

The neuronal activities of the VTA neurons during optical stimulations were electrically recorded in vivo via the implanted optrode array devices. Figures 1(e) and 1(f) showed the spike firing activities and local field potentials recorded from multiple channels in the VTA region, respectively. The laser bars displayed in both Figures 1(e) and 1(f) indicated $473 \mathrm{~nm}$ laser delivery with the same stimulating pattern as in the free-roaming tasks (see Section 2.4.1). It was observed that a 1.0-second laser stimulus induced a significant increase in spike firing activities reflected in most channels (13 out of 16) from the array device, and after the end of laser stimulus the spike activity fell to the basic level. Similar results were observed in LFP changes where the field potentials exhibited a higher magnitude during the $1.0 \mathrm{~s}$ laser stimulus. Both results indicated that the in vivo optical stimulations on the optogenetic neurons enabled an increased neuronal activity in the VTA region.

3.2. The Optogenetic Rats Exhibited an Increased Locomotor Activity upon Light Stimulation during Free-Roaming Tasks. The optogenetic rats were performed with free-roaming behavioral tasks to illustrate the influence of optical stimulation on their locomotor activities. Two typical tracking maps of the rat in-field roaming during a nonstim and an optic stim session, respectively, were shown in Figures 2(a) and 2(b). Normally in the nonstim session the rat would exhibit a short-term roaming for exploration and then stay still against the edge wall for the rest of the session. While, in the optic stim session, the rat exhibited a more intense and long-term free roaming that it would travel more laps along the circular field. Also it was observed that during the optic stim session the rats took far more approaches into the center region of the field which is considered as an open field where normal rats seldom stay [27]. The time courses of the intrasession distances revealed statistical differences between optic stim and nonstim sessions for most days of free-roaming tasks (see Figure 2(d)). The bar plot of average intrasession distances across the six task days further indicated that the rats during optic stim sessions ran a significantly higher distance over nonstim sessions (see Figure 2(e)). The above data suggested that the rats under the optogenetic stimulation in VTA were able to exhibit a higher locomotor activity in the free-roaming tasks.

Although the rats had shown an increased locomotor activity in the above tasks, one might doubt that the "light leakage" during laser stimulations would influence on the behaving rats by light flashings over the rat eyes. An alternative series of free-roaming sessions with the laser blocked at the FC interface over the rat head (denoted optic blocking) was designed to eliminate the "light leakage" influences. Figure 2(c) displayed the tracking map from a typical optic blocking session. Compared with Figure 2(a) from the nonstim session, little change was observed either on traveling laps or on approaches into the center field. The time courses of optic blocking sessions versus nonstim sessions revealed no statistical differences for the task days (see Figure 2(d)), and nor did the average bar plot show differences (see Figure 2(e)). Interestingly, it was observed from the plots that the optic blocking sessions held a slightly higher locomotion than the nonstim ones, yet with no statistical changes. This phenomenon was probably related to certain baseline properties of the rats' behavioral conditions, such as the curiosity to or being frightened with the frequent light flashings that we usually observed at the beginning of the free-roaming tasks. In general the data above suggested that simple light flashing over the rat eyes had no significant influences on the locomotor activity of the free behaving optogenetic rats. Totally, the behavioral data of free-roaming studies shown in Figure 2 supported our hypothesis that optogenetic activation of the excitatory neurons expressing CaMKII $\alpha$ in the VTA has positive reinforcement properties on the locomotor activity of free behaving rats.

\subsection{Optogenetic Rats Achieved Better Performances in Lever-} Pressing Tasks That Involves Reward Learning. The underlying mechanisms of the optogenetic activation in the freeroaming tasks were probably depending on the reinforcing properties of VTA in the process of reward learning. Thereby we hypothesized that the optogenetic excitation of the VTA neurons expressing CaMKII $\alpha$ played a role of "virtual reward" that could induce reward seeking behaviors. For further investigation we conducted a set of lever-pressing tasks with optical self-stimulation in the VTA region for both optogenetic rats and controls as mentioned in Section 2.4.1. The data plotted in Figures 3(a) and 3(b) showed that the total intrasession lever presses of optogenetic rats were significantly higher than those of controls in most sessions. Also we note that the time course of optogenetic rats revealed an ascending tendency over the sessions. Besides, the plots of "correct rate" indicating the percentage of active lever presses during each session (see Figures 3(c) and 3(d)) showed that the optogenetic rats held a significantly higher correct rate than the controls for most sessions. Likewise, the time course of optogenetic rats in Figure 3(c) revealed an ascending tendency, whereas the course of controls appeared random with no tendency at all. In total, the data of lever-pressing tasks indicated that the optogenetic rats tend to make far more lever presses than the controls for obtaining the optical stimulation. Since the lever-pressing study is considered as a typical test for validation of reward learning, the results from the above data provided evidence to our hypothesis that the optogenetic activation of the excitatory neurons expressing CaMKII $\alpha$ in VTA played a role of virtual reward that led to a positive effect on the reward learning processes.

Besides the learning of lever pressing, the learning of free roaming for obtaining the optical "reward" was also behaviorally conditioned on the optogenetic rats in this study. 


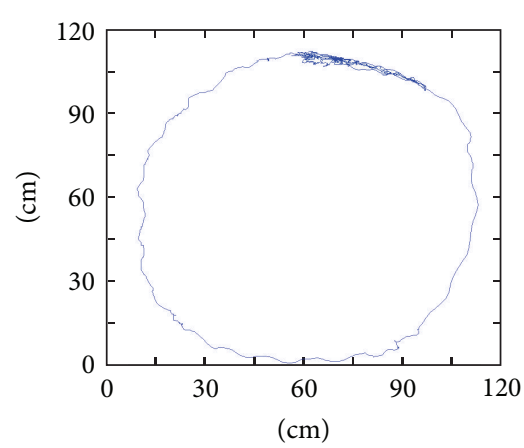

(a)

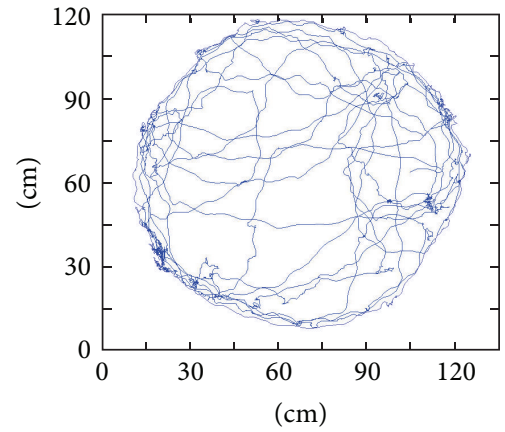

(b)

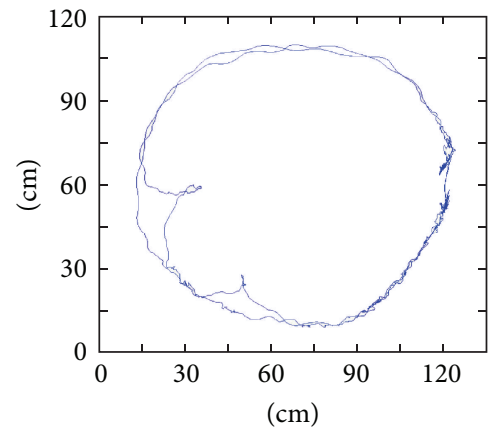

(c)
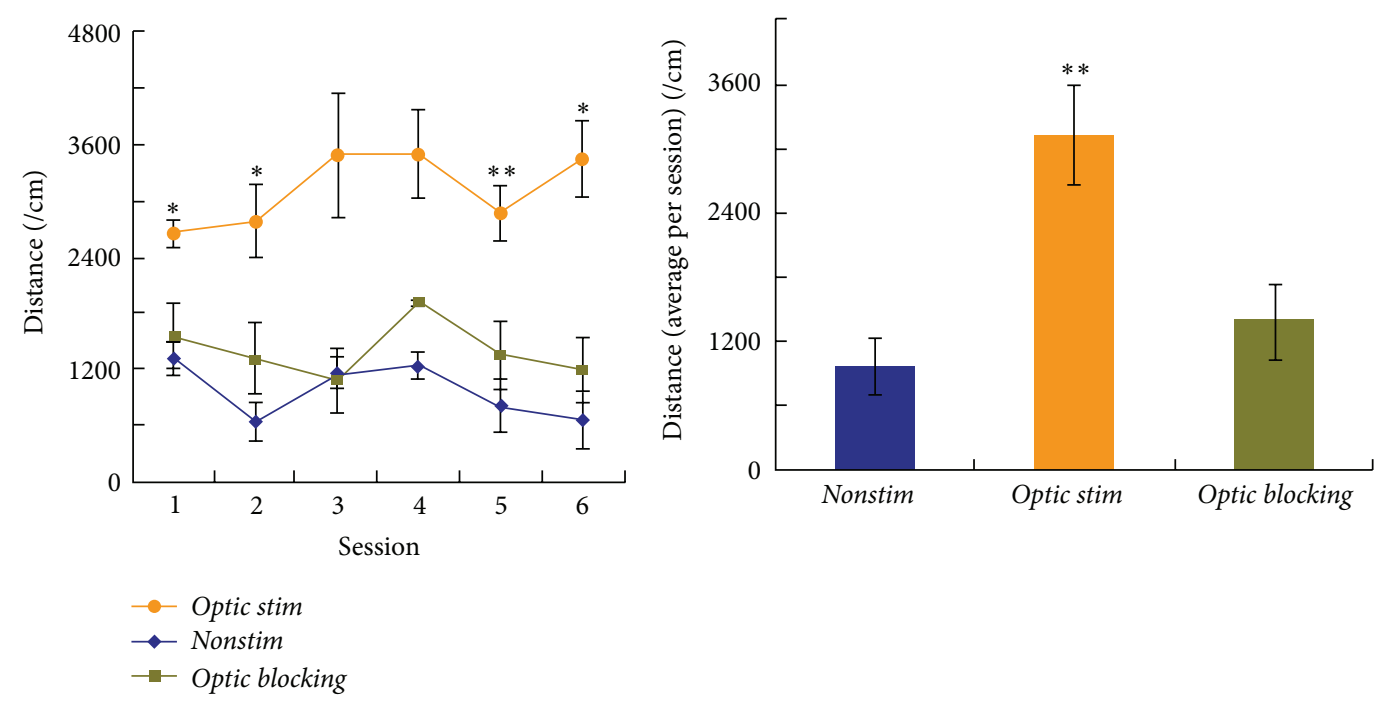

(d)

(e)

FIGURE 2: Data of the free-roaming tasks between the nonstim, optic stim, and optic blocking sessions. ((a)-(c)) Field tracking maps typically for the nonstim, the optic stim, and the optic blocking session, respectively. (d) Time courses of the intrasession distances for the above three sessions (see the figure legends). The error bar in each session dot represents the standard deviation of all the rats for the session $(n=6$ for each session dot). Single asteroids represent statistical differences between the session dots of optic stim and nonstim, whereas double asteroids represent significant differences between them. (e) The results from the time course (d) were averaged and then bar-plotted over the three sessions. Double asteroids represent a significant difference compared to the results of nonstim sessions.

The procedures of training were basically the same with previous free-roaming tasks except that the laser stimulations were given manually once the rat started roaming. After six days of training, we repeated the free-roaming tasks on these trained rats over both optic stim (or the trained stim) and nonstim sessions. From the tracking maps (Figures 4(a) and 4(b)) a far more intense roaming in the trained stim session than the nonstim one appeared. Moreover, the time course of the trained stim sessions displayed a significant higher level over the nonstim one (see Figure 4(c)). Besides, a clear view of ascending tendency was observed from the trained stim course, which probably indicated the enhancement of reward learning processes of the rats. Both bar plots (Figures 4(d) and $4(\mathrm{e}))$ revealed that the average intrasession locomotion in the trained stim sessions was significantly higher than the nonstim ones and appeared the greatest among all the four types of sessions (nonstim, optic blocking, optic stim, and trained stim). Remarkably, the results of the trained stim sessions were statistically higher than those of the optic stim sessions before training, consistent with the properties of reward learning behaviors. Taken together, the above results suggested that reward learning took part in the mechanism underlying the optogenetic activation of the neurons expressing CaMKII $\alpha$ in the VTA of behaving rats and that by the reinforcement of reward learning, better performances on the rat locomotor activity were likely to be achieved.

3.4. The VTA Neurons under the Optogenetic Activation Were Partly Dopaminergic. In the above experiments we employed optogenetic manipulations on the excitatory neurons in the rat VTA region and conditioned a higher locomotor activity of free behaving rats based on the mechanism of reward learning. It is usually considered that dopaminergic (DA) neurons in the ventral striatum mediate in the reward learning mechanisms. Thus we investigated whether the DA neurons in VTA were selectively activated by the optogenetic manipulations in our study. The immunohistochemistry studies were employed to assess the locations of both the cell 


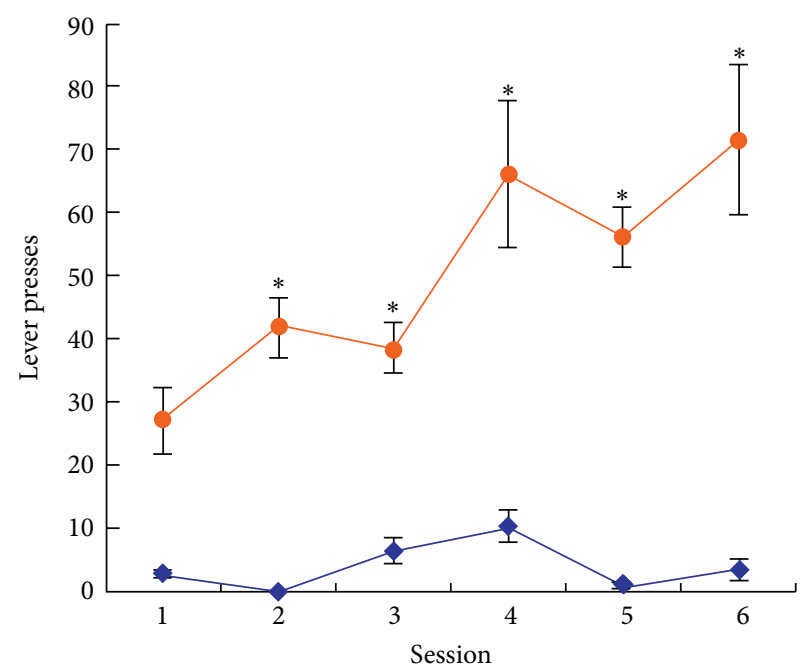

(a)

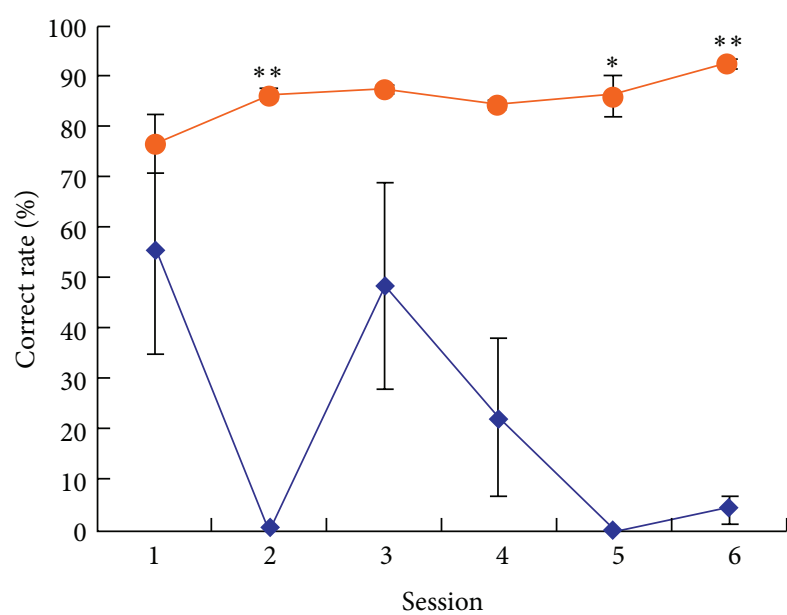

(c)

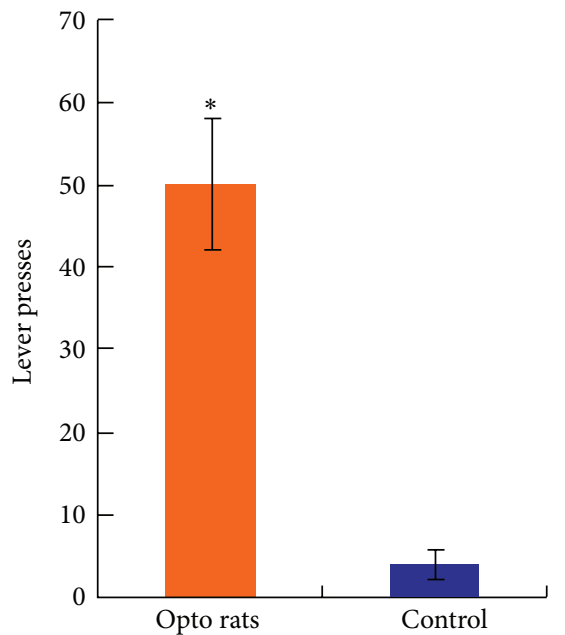

(b)

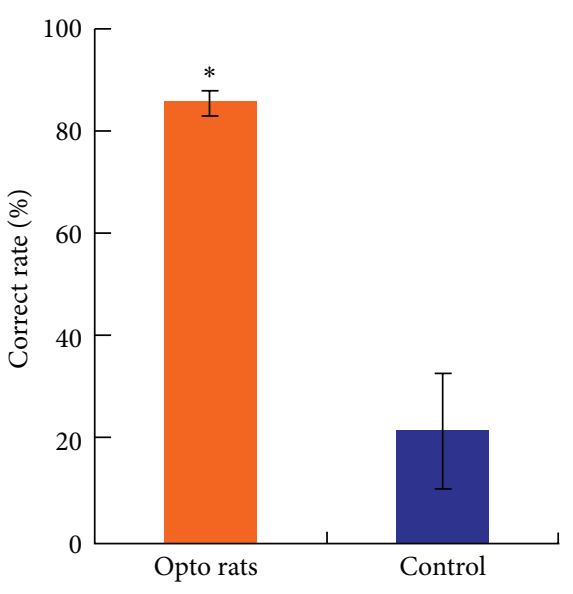

(d)

Figure 3: Data of the lever-pressing tasks for both optogenetic rats (denoted "opto rats") and control rats. (a) Time courses of the total lever presses intrasession for opto rats and control rats individually (see the figure legends). (b) The total lever presses from (a) were averaged across all the six sessions and bar-plotted. (c) The correct rates, indicating the percentages of active lever presses within single sessions, were plotted as time courses for opto rats and control rats (see the figure legends). (d) Bar plot of the correct rates calculated from a total collection of lever-pressing data. For all the subfigures, the error bars in session dots or in bars represent the standard deviation of all the rats undertaking the session ( $n=6$ for each session dot or bar). Single asteroids represent statistical differences between the opto rats and controls, whereas double asteroids represent significant differences between them.

bodies labeling tyrosine hydroxylase- (TH-) GFP, a marker for DA neurons, and the cell bodies expressing CaMKII $\alpha$ mCherry by optogenetic transduction in the VTA region. Figure 5 displayed a typical set of the results. Figures 5(a)5 (c) revealed that both the expressions of CaMKII $\alpha$-mCherry and TH-GFP were distributed in relative narrow area that contains the VTA. It was observed that the TH positive neurons appeared a higher density than the neurons expressing CaMKII $\alpha$, consistent with previous reports that DA neurons held a predominant percentage in VTA [22]. Both the expressions of CaMKII $\alpha$-mCherry and TH-GFP were ranged over the VTA region. Figures 5(d)-5(f) revealed that most $\mathrm{TH}$ positive neurons and CaMKII $\alpha$ positive neurons differed in cell morphologies. However, despite the fact that most
TH positive neurons do not express CaMKII $\alpha$, and many CaMKII $\alpha$ positive ones do not contain TH [19], there existed a substantial number of neurons expressing both CaMKII and TH (see Figure 5(f) and white arrows in Figure 5(i)). The immunohistochemistry data above indicated that part of the VTA neurons expressing CaMKII $\alpha$ corelease TH, thus exhibiting a dopaminergic property. These "dopaminergic" neurons appeared to take only a small percentage of the vast DA neurons within the VTA region.

\section{Discussion}

In this study we employed in vivo optogenetic transduction on the excitatory neurons in the brain region of VTA for 


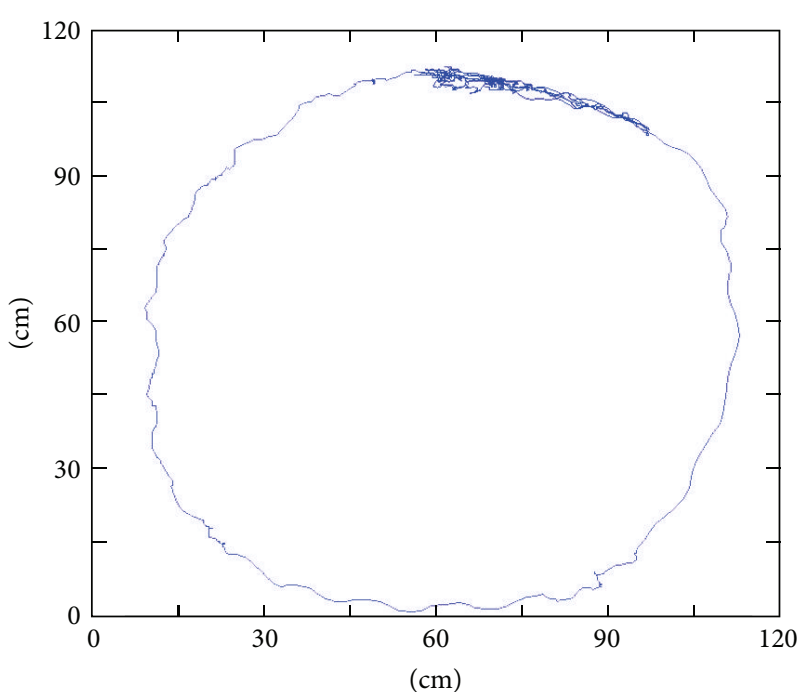

(a)

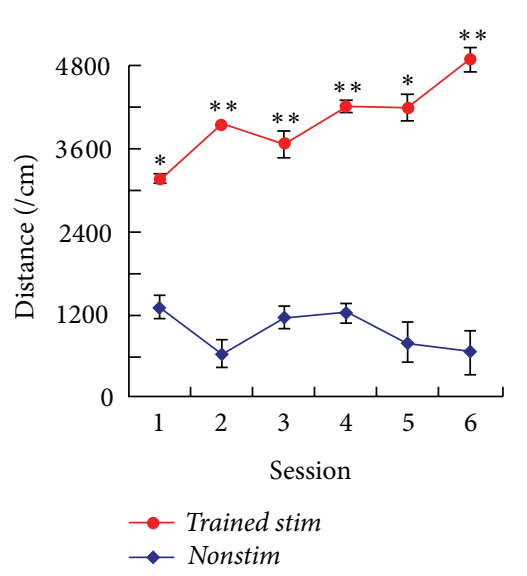

(c)

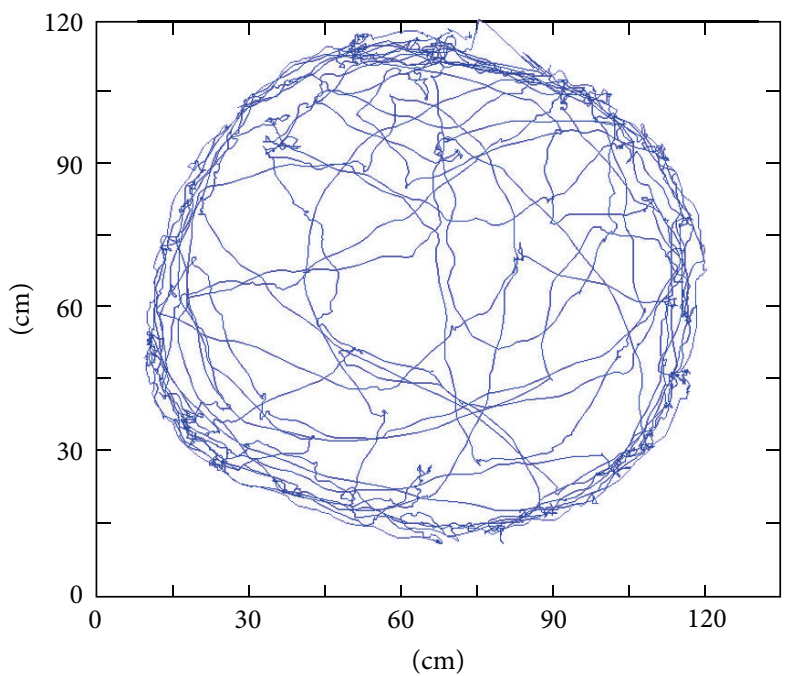

(b)

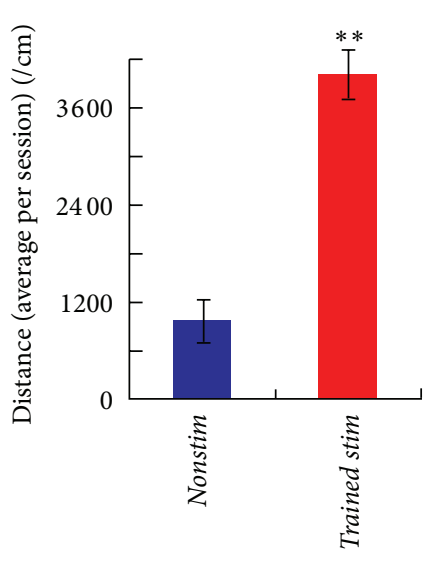

(d)

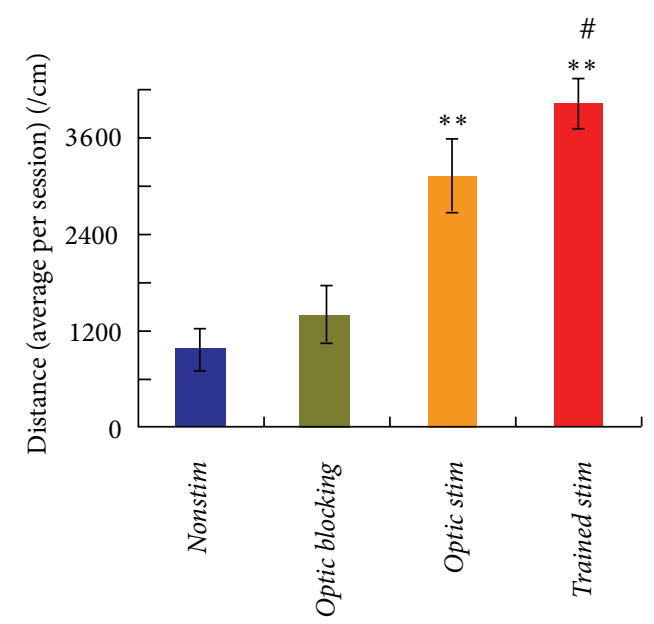

(e)

Figure 4: Data of free-roaming tasks combined with the trained stim sessions. (a and b) Field tracking maps of the nonstim and the trained stim session, respectively. Data and figures were acquired in the same manner as in Figure 2. (c) Time courses of the intrasession distances for both the trained stim and the nonstim sessions (see the figure legends). The error bar in each session dot represents the standard deviation of all the rats for the session ( $n=6$ for each session dot). Double asteroids represent significant differences between the session dots of trained stim and nonstim. (d) The intrasession distances were averaged from (c) and were bar-plotted over the trained stim and the no-stim sessions, where double asteroids represent a significant difference between them. (e) The bar plot of the average intrasession distances for all the four types of free-roaming sessions, the nonstim, the optic blocking, the optic stim, and the trained stim. Double asteroids represent significant differences compared to the nonstim data, while the sharp represents a statistical difference between the optic stim and the trained stim sessions.

adult rats by viral delivery of the AAV vector carrying ChR2 opsin genes. Our findings on the free-roaming tasks demonstrated that the optogenetic rats with ChR2 expression in the excitatory neurons expressing CaMKII $\alpha$ in VTA behaved at an increased locomotor activity upon $473 \mathrm{~nm}$ light activation while freely roaming in the circular field. This phenomenon was not likely owing to simple "light leak" effects with the blue light emitting over the rat eyes.

Further results on the lever-pressing tasks revealed that reward learning was mediating in the mechanisms underlying the CaMKII optogenetic activation in VTA in the above studies. These findings were consistent with previous reports that the activation of VTA neurons was able to induce reward learning behaviors for receiving the intracranial selfstimulations (ICSSs) [28]. Remarkably, in previous studies the highly frequent lever-pressing behaviors were typically induced by electrical stimulations, during which all the neural populations within the VTA were activated compulsively, making it unclear whether any type of subpopulations was involved in the reward learning behaviors. In this paper, however, we specifically activated the excitatory neurons expressing CaMKII $\alpha$ in the VTA by optogenetic manipulations and induced similar lever-pressing behaviors of the rats. These results indicated that these excitatory neurons 


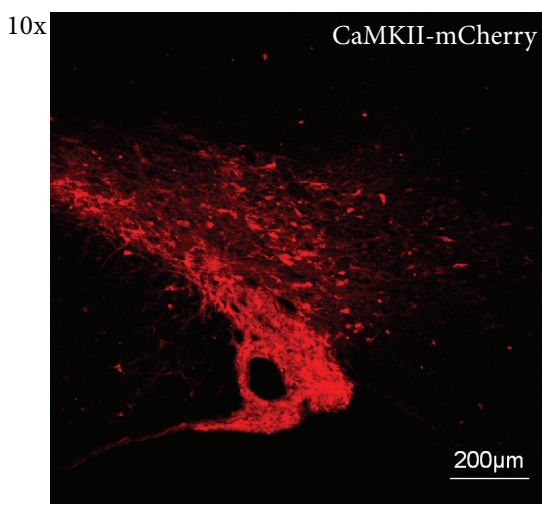

(a)

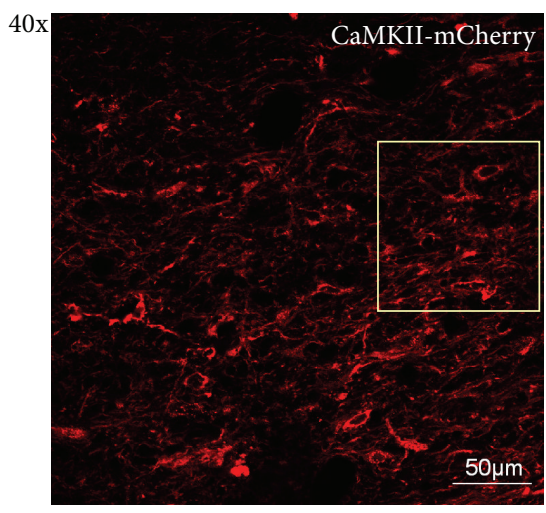

(d)

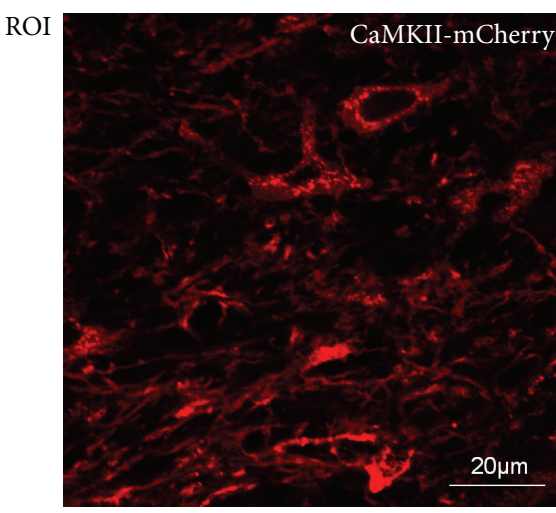

(g)

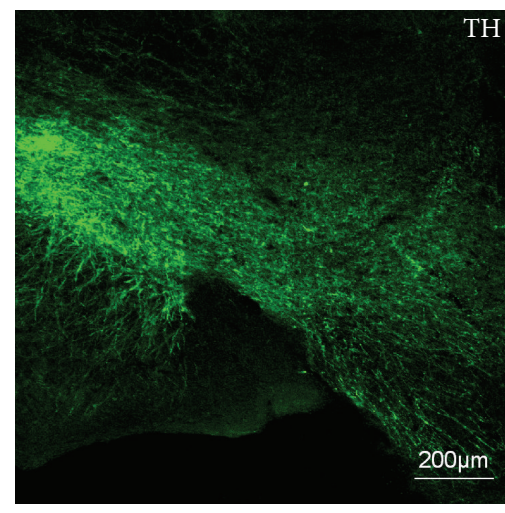

(b)

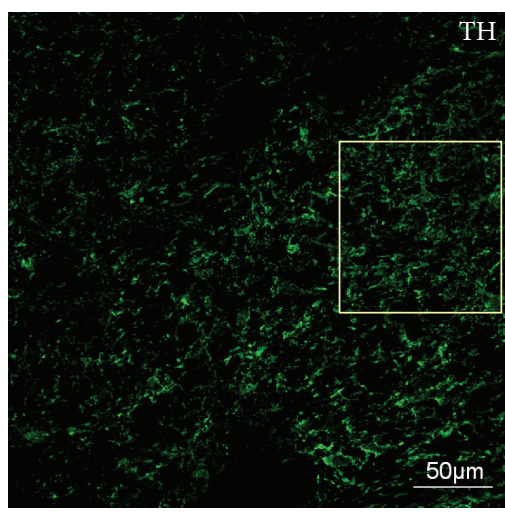

(e)

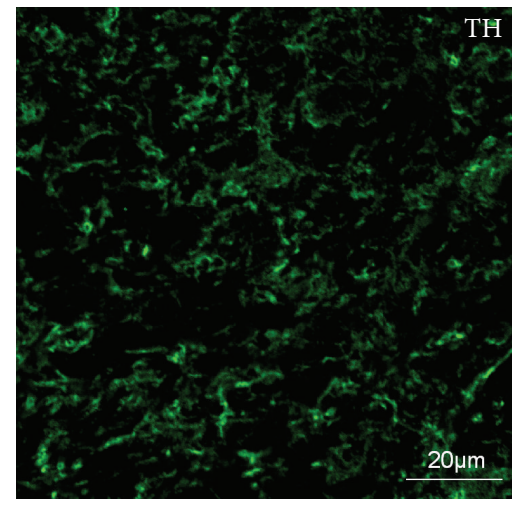

(h)

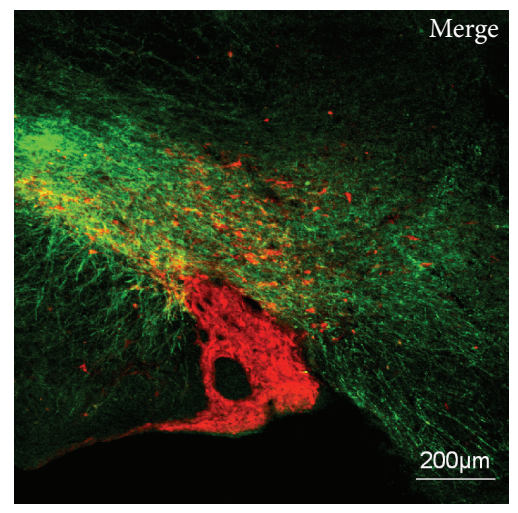

(c)

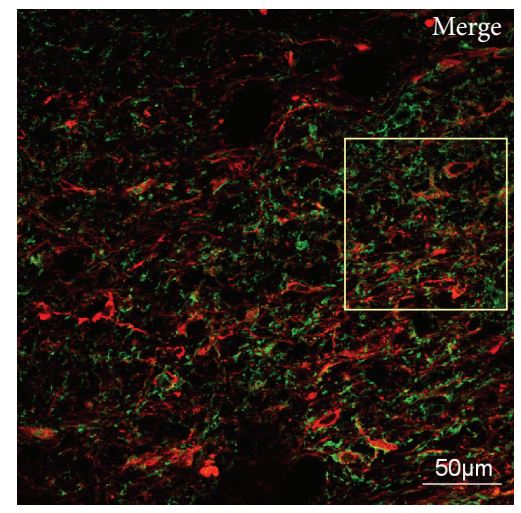

(f)

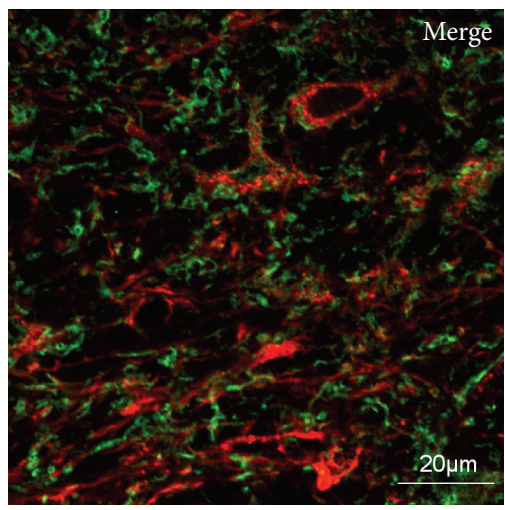

(i)

FIGURE 5: Immunochemistry results from the VTA region of brain slices from the optogenetic rats. All the subfigures were photographed under confocal microscopy and processed using the FV1000 software (Olympus Inc., Japan). The red channels represent CaMKII $\alpha$-mCherry expressions and the green channels represent TH expressions, whereas the right column (Figures 5(c), 5(f), and 5(i)) indicates the merged two channels. ((a)-(c)) A typical view of the CaMKII $\alpha$-mCherry and TH-GFP expressions under 10x microscopy. ((d)-(f)) A selected VTA region observed under 40x microscopy where the CaMKII $\alpha$ and TH expressions overlapped. ((g)-(i)) A region of interest (ROI) from the rectangular window shown in ((d)-(f)). The white arrows in (i) point out the several neuronal cell bodies merged with red and green channels, indicating that these neurons coexpress CaMKII $\alpha$ and TH.

expressing CaMKII $\alpha$ were probably a key population in the VTA that played a vital role in the conditioning of reward learning as in the lever-pressing tasks. Moreover, we repeated the free-roaming tasks on the optogenetic rats after a shortterm training where light stimuli were given, while the rat was roaming forward, and observed that the rats performed a higher locomotor activity than before. These results further indicated that the reinforcement of reward learning placed a positive effect on the results of free-roaming tasks.

The immunohistochemistry further explored the nature of the excitatory neurons expressing CaMKII $\alpha$ in VTA under the optogenetic manipulation. The results revealed that those neurons expressing CaMKII $\alpha$ were partly collocated with dopaminergic neurons at the VTA region. The "collocated 
neurons" took only a small percentage of the VTA DA neurons, but they were observed in an appropriate area where light stimulations were reachable, which suggested that these neurons coexpressing CaMKII $\alpha$ and DA were activated upon light stimulations and probably took an active part in the behavioral conditionings in this study. Since the expressions of CaMKII $\alpha$ were widely implicated in glutamatergic neurons [1], those "collocated neurons" were likely to be neurons that corelease DA and glutamine in nature, consistent with previous reports that there exist a substantial area of excitatory neurons in the VTA region that corelease dopamine and glutamine and contribute to the functioning of reward learning and related behavioral conditionings $[29,30]$.

However, it was suggested from the immunohistochemistry results that there existed an alternative type of neurons that expresses CaMKII $\alpha$ but does not express TH. These neurons differed in cell morphology from the dopaminergic neurons and exhibited an expression of CaMKII $\alpha$-mCherry. Based on the results above, it is inferred that those nondopaminergic neurons were probably simple glutamatergic neurons, consistent with previous reports that glutamatergic neurons were present in the VTA $[19,31]$. According to previous reports, the exists a type of glutamatergic neurons in the VTA region that release VGluT2 but do not release dopamine, and they exhibit different electrophysiological properties and neuronal projections apart from the VTA neurons coexpressing glutamine and dopamine [32]. Moreover, these nondopaminergic neurons expressing CaMKII $\alpha$ were also located in VTA within the region of light activation, which indicated that these neurons were likely to take part in the optogenetic activations as well. Combined together, the excitatory neurons expressing CaMKII $\alpha$ in the VTA under optogenetic activations were composed of different types of neuronal subpopulation: one is the dopaminergic neurons that may corelease glutamine, and the other neurons were probably purely glutamatergic. Both types of neurons were likely to play vital roles in the behavioral conditionings as we conducted in this paper.

In conclusion, we employed optogenetic manipulations for free behaving animals based on viral transduction of ChR2 in the brain region of VTA and behavioral conditionings on reward learning. The results demonstrated that optogenetic activations of the excitatory neurons expressing CaMKII $\alpha$ in the VTA were able to upregulate the locomotor activity of free behaving rats and that by reinforcement of the reward learning behaviors the rats' locomotor activities were getting even enhanced. Further immunohistochemistry results revealed the nature of these VTA neurons expressing CaMKII $\alpha$, which were partly dopaminergic that may play a vital role in the above behavioral conditionings. Future work may include further investigations on the nature of these neurons under the optogenetic activation in this study, such as the relationships between the neurons expressing CaMKII $\alpha$ and glutamatergic neurons in the VTA. Moreover, this study has provided a novel method to upregulate the locomotor activity by inducing the reward seeking behavior and by reward learning the "reward-motion" connectivity had gotten enhanced for the optogenetic rats, thus indicating further applications in the field of neural engineering such as animal robotics for precise control of animal behaviors.

\section{Conflict of Interests}

The authors declare that there is no conflict of interests regarding the publication of this paper.

\section{Acknowledgments}

The work of this paper was supported by Grants from the National Basic Research Program of China (2011CB504405), the National High Technology Research and Development Program of China (2012AA011602), the National Natural Science Foundation (NSF) of China (61233015), the National NSF of China (61103107; 61001172, 61003150, 61305145, 61305146, and 31371001), the Specialized Research Fund for the Doctoral Program of Higher Education (20100101120104, 20100101120154, and 20100101120166), the Fundamental Research Funds for the Central Universities, and the Zhejiang provincial international science and technology cooperation program (2012C24025). The authors would thank Chaonan Yu for providing technical support on the fabrication of the optrode array device and on part of the surgery of craniotomy. Also the authors thank Professor Shumin Duan, Dr. Rui Zhou, and Dr. Hongbin Yang for providing tutorials on the immunohistochemistry studies in this paper.

\section{References}

[1] O. Yizhar, L. E. Fenno, T. J. Davidson, M. Mogri, and K. Deisseroth, "Optogenetics in Neural Systems," Neuron, vol. 71, no. 1, pp. 9-34, 2011.

[2] K. Deisseroth, G. Feng, A. K. Majewska, G. Miesenböck, A. Ting, and M. J. Schnitzer, "Next-generation optical technologies for illuminating genetically targeted brain circuits," Journal of Neuroscience, vol. 26, no. 41, pp. 10380-10386, 2006.

[3] I. B. Witten, E. E. Steinberg, S. Y. Lee et al., "Recombinasedriver rat lines: tools, techniques, and optogenetic application to dopamine-mediated reinforcement," Neuron, vol. 72, no. 5, pp. 721-733, 2011.

[4] K. M. Tye and K. Deisseroth, "Optogenetic investigation of neural circuits underlying brain disease in animal models," Nature Reviews Neuroscience, vol. 13, no. 4, pp. 251-266, 2012.

[5] K. Zalocusky and K. Deisseroth, "Optogenetics in the behaving rat: integration of diverse new technologies in a vital animal model," Optogenetics, vol. 1, pp. 1-17, 2013.

[6] V. Gradinaru, K. R. Thompson, F. Zhang et al., "Targeting and readout strategies for fast optical neural control in vitro and in vivo," Journal of Neuroscience, vol. 27, no. 52, pp. 14231-14238, 2007.

[7] A. M. Aravanis, L.-P. Wang, F. Zhang et al., "An optical neural interface: in vivo control of rodent motor cortex with integrated fiberoptic and optogenetic technology," Journal of Neural Engineering, vol. 4, no. 3, pp. S143-S156, 2007.

[8] T. Tokuda, S. Nakajima, Y. Maezawa et al., "An in vitro demonstration of CMOS-based optoelectronic neural interface device for optogenetics," in Proceedings of the 35th Annual 
International Conference of the IEEE Engineering in Medicine and Biology Society (EMBS '13), pp. 799-802, Osaka, Japan, 2013.

[9] K. Deisseroth, “Optogenetics," Nature Methods, vol. 8, no. 1, pp. 26-29, 2011.

[10] F. Zhang, L.-P. Wang, E. S. Boyden, and K. Deisseroth, "Channelrhodopsin-2 and optical control of excitable cells," Nature Methods, vol. 3, no. 10, pp. 785-792, 2006.

[11] J. A. Cardin, M. Carlén, K. Meletis et al., “Targeted optogenetic stimulation and recording of neurons in vivo using cell-typespecific expression of Channelrhodopsin-2," Nature Protocols, vol. 5, no. 2, pp. 247-254, 2010.

[12] H. Wang, J. Peca, M. Matsuzaki et al., "High-speed mapping of synaptic connectivity using photostimulation in Channelrhodopsin-2 transgenic mice," Proceedings of the National Academy of Sciences of the United States of America, vol. 104, no. 19, pp. 8143-8148, 2007.

[13] J. T. Ting and G. Feng, "Development of transgenic animals for optogenetic manipulation of mammalian nervous system function: progress and prospects for behavioral neuroscience," Behavioural Brain Research, vol. 255, pp. 3-18, 2013.

[14] Y.-P. Zhang, N. Holbro, and T. G. Oertner, "Optical induction of plasticity at single synapses reveals input-specific accumulation of $\alpha$ CaMKII," Proceedings of the National Academy of Sciences of the United States of America, vol. 105, no. 33, pp. 12039-12044, 2008.

[15] M. Papa, L. Diewald, M. P. Carey, F. J. Esposito, U. A. Gironi Carnevale, and A. G. Sadile, "A rostro-caudal dissociation in the dorsal and ventral striatum of the juvenile SHR suggests an anterior hypo- and a posterior hyperfunctioning mesocorticolimbic system," Behavioural Brain Research, vol. 130, no. 1-2, pp. 171-179, 2002.

[16] B. J. Wiltgen, M. Law, S. Ostlund, M. Mayford, and B. W. Balleine, "The influence of Pavlovian cues on instrumental performance is mediated by CaMKII activity in the striatum," European Journal of Neuroscience, vol. 25, no. 8, pp. 2491-2497, 2007.

[17] S. K. Talwar, S. Xu, E. S. Hawley, S. A. Weiss, K. A. Moxon, and J. K. Chapin, "Rat navigation guided by remote control," Nature, vol. 417, no. 6884, pp. 37-38, 2002.

[18] Z.-Y. Feng, W.-D. Chen, X.-S. Ye et al., "A remote control training system for rat navigation in complicated environment," Journal of Zhejiang University: Science A, vol. 8, no. 2, pp. 323330, 2007.

[19] T. Yamaguchi, W. Sheen, and M. Morales, "Glutamatergic neurons are present in the rat ventral tegmental area," European Journal of Neuroscience, vol. 25, no. 1, pp. 106-118, 2007.

[20] L. W. Swanson, “The projections of the ventral tegmental area and adjacent regions: a combined fluorescent retrograde tracer and immunofluorescence study in the rat," Brain Research Bulletin, vol. 9, no. 1-6, pp. 321-353, 1982.

[21] R. A. Wise, "Brain reward circuitry: insights from unsensed incentives," Neuron, vol. 36, no. 2, pp. 229-240, 2002.

[22] H. L. Fields, G. O. Hjelmstad, E. B. Margolis, and S. M. Nicola, "Ventral tegmental area neurons in learned appetitive behavior and positive reinforcement," Annual Review of Neuroscience, vol. 30, pp. 289-316, 2007.

[23] K. Schierberl, J. Hao, T. F. Tropea et al., "Ca 1.2 l-type $\mathrm{Ca}^{2+}$ channels mediate cocaine-induced glual trafficking in the nucleus accumbens, a long-term adaptation dependent on ventral tegmental area ca 1.3 channels," Journal of Neuroscience, vol. 31, no. 38, pp. 13562-13575, 2011.
[24] J. D. Lenz and M. K. Lobo, "Optogenetic insights into striatal function and behavior," Behavioural Brain Research, vol. 255, pp. 44-54, 2013.

[25] S. Guo, H. Zhou, J. Zhang, K. Xu, and X. Zheng, "A multielectrode array coupled with fiberoptic for deep-brain optical neuromodulation and electrical recording," in Proceedings of the 35th Annual International Conference of the IEEE Engineering in Medicine and Biology Society (EMBS '13), pp. 2752-2755, Osaka, Japan, 2013.

[26] C. Sun et al., "Automatic navigation for rat-robots: ubiquitous computing in animals," Journal of Bionic Engineering, vol. 10, no. 1, pp. 46-56, 2013.

[27] R. N. Walsh and R. A. Cummins, “The open-field test: a critical review," Psychological Bulletin, vol. 83, no. 3, pp. 482-504, 1976.

[28] J. F. Cheer, B. J. Aragona, M. L. A. V. Heien, A. T. Seipel, R. M. Carelli, and R. M. Wightman, "Coordinated accumbal dopamine release and neural activity drive goal-directed behavior," Neuron, vol. 54, no. 2, pp. 237-244, 2007.

[29] N. Chuhma, H. Zhang, J. Masson et al., "Dopamine neurons mediate a fast excitatory signal via their glutamatergic synapses," Journal of Neuroscience, vol. 24, no. 4, pp. 972-981, 2004.

[30] H.-C. Tsai, F. Zhang, A. Adamantidis et al., "Phasic firing in dopaminergic neurons is sufficient for behavioral conditioning," Science, vol. 324, no. 5930, pp. 1080-1084, 2009.

[31] A. Dobi, E. B. Margolis, H.-L. Wang, B. K. Harvey, and M. Morales, "Glutamatergic and nonglutamatergic neurons of the ventral tegmental area establish local synaptic contacts with dopaminergic and nondopaminergic neurons," Journal of Neuroscience, vol. 30, no. 1, pp. 218-229, 2010.

[32] T. S. Hnasko, G. O. Hjelmstad, H. L. Fields, and R. H. Edwards, "Ventral tegmental area glutamate neurons: electrophysiological properties and projections," The Journal of Neuroscience, vol. 32, no. 43, pp. 15076-15085, 2012. 


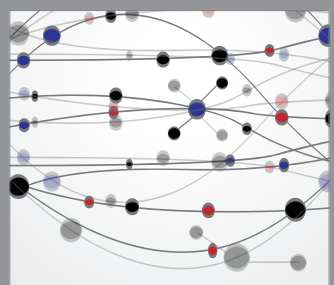

The Scientific World Journal
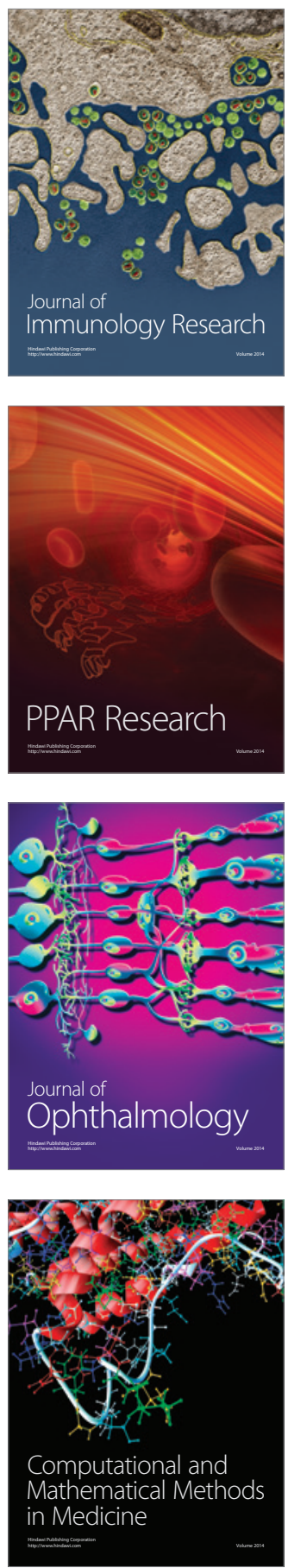

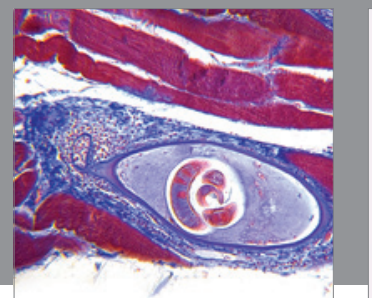

Gastroenterology

Research and Practice
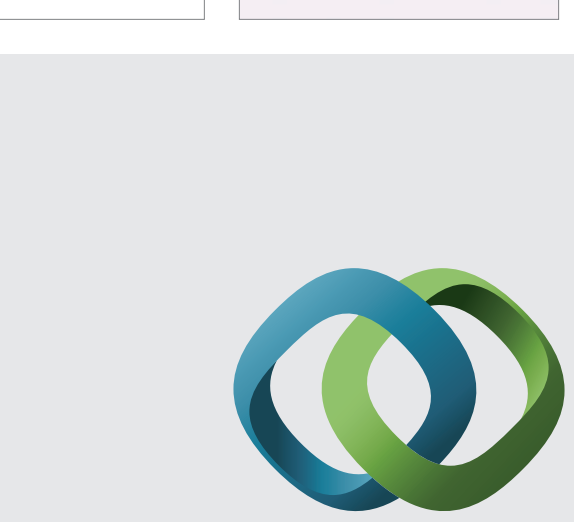

\section{Hindawi}

Submit your manuscripts at

http://www.hindawi.com
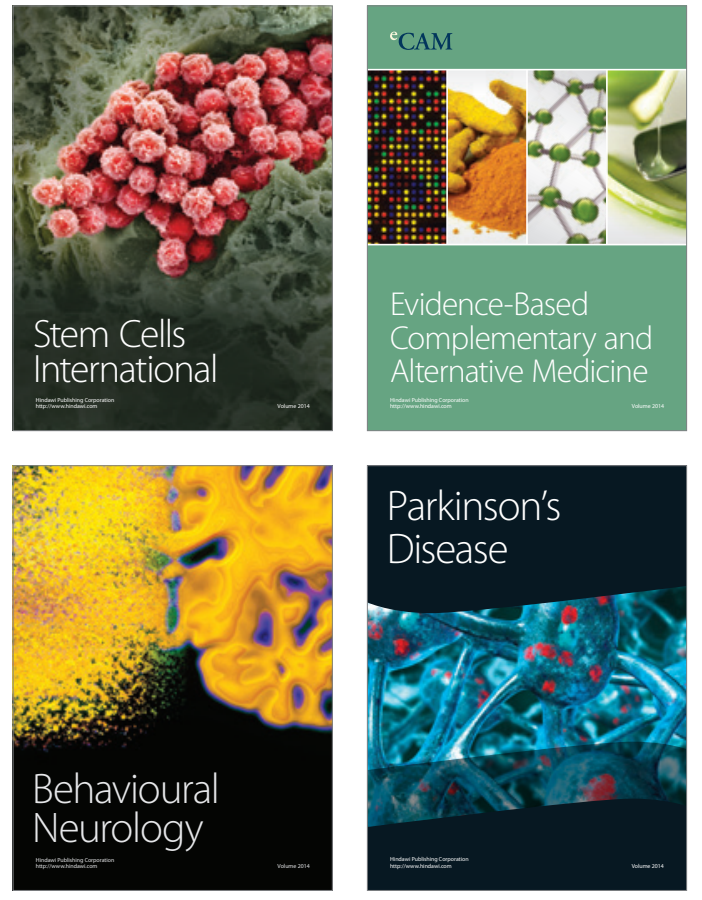
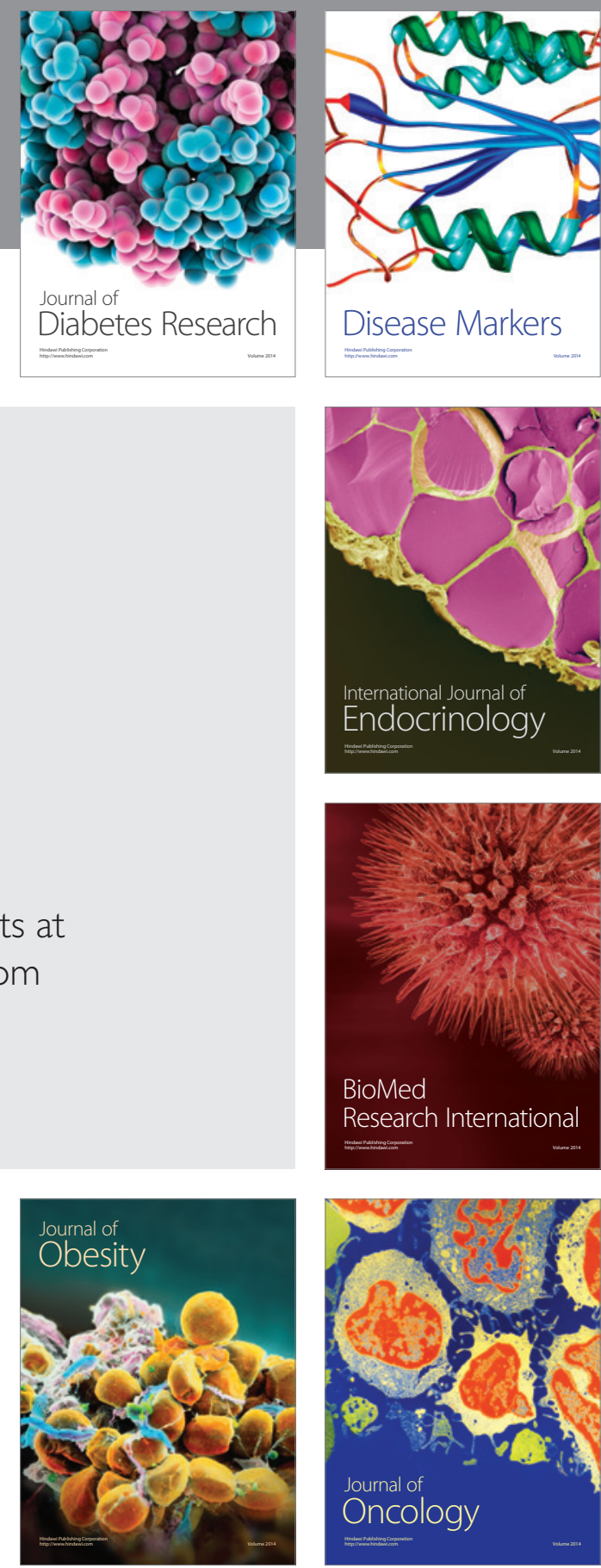

Disease Markers
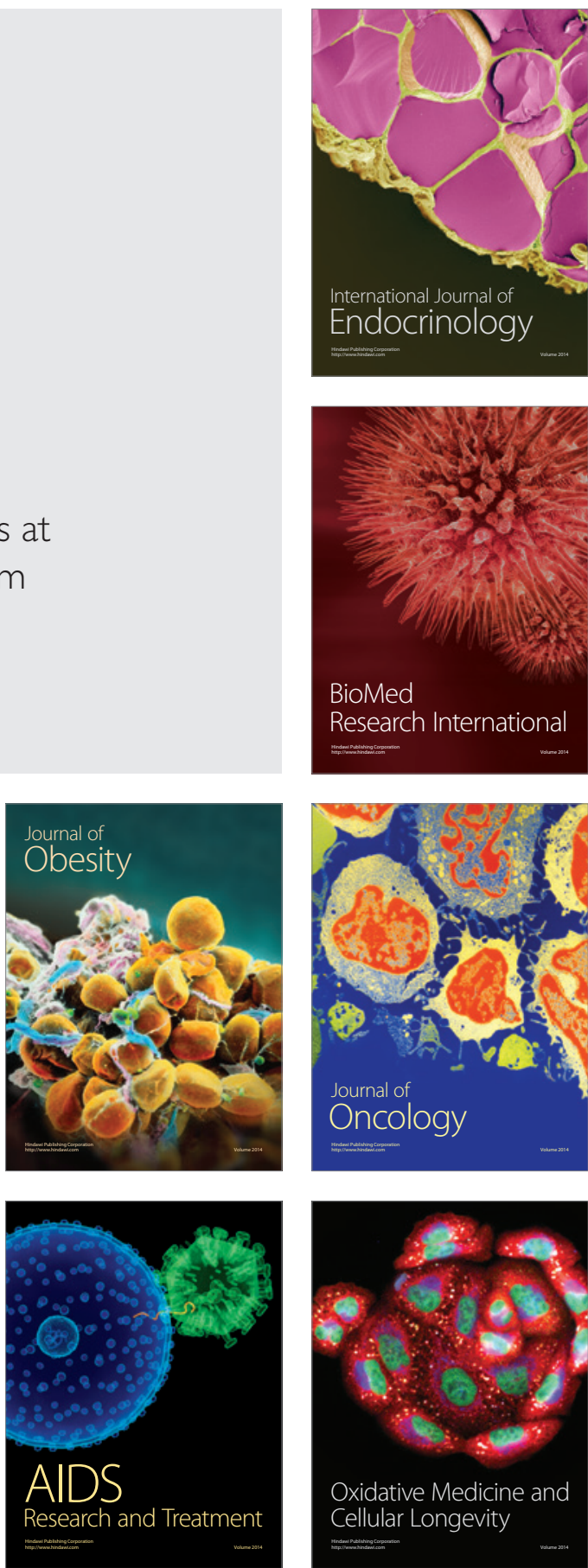